\title{
PRODUCTION OF HELIUM IN MASSIVE OBJECTS*
}

\author{
ROBERT V. WAGONER ** \\ Dept. of Astronomy and Center for Radiophysics and Space Research, \\ Cornell University, Ithaca, N.Y. 14850, U.S.A.
}

\begin{abstract}
The production of helium within supermassive stars, supermassive disks, and 'little bangs' is discussed. The conclusions are summarized at the end of the paper.
\end{abstract}

\section{Introduction}

The bulk of the helium observed throughout the universe has most likely been produced by one of two fundamental processes. These are (1) a slow conversion of protons to ${ }^{4} \mathrm{He}$ during the quasi-static evolution of some object, followed by an expulsion of the helium-rich material before further evolution has burned the helium, or (2) a rapid expansion from very high temperatures $\left(>10^{10} \mathrm{~K}\right)$, where nucleons are again available for conversion to ${ }^{4} \mathrm{He}$ as the gas cools. For the most part, we will not be concerned with the abundance of ${ }^{3} \mathrm{He}$.

Dr. Kippenhahn has described what contribution ordinary stars can make, mainly through process 1 . Dr. Novikov will describe what contribution the universal bigbang can make through process 2 . In this talk we shall consider the possible outcome of these processes operating within massive objects, which we define somewhat arbitrarily to be bodies of mass $10^{4} M_{\odot} \lesssim M \lessgtr 10^{12} M_{\odot}$ in which radiation pressure dominates. In connection with process 1 we shall consider two types of objects: (a) supermassive stars, introduced by Hoyle and Fowler (1963a, b), and (b) supermassive disks, whose properties have recently been studied by J. Bardeen, E. Salpeter and myself (Bardeen and Wagoner, 1969; Salpeter and Wagoner, 1971; Wagoner and Salpeter, 1970). We shall mostly draw qualitative conclusions about the amount of helium produced in such objects. On the other hand, the operation of process 2 within a massive object, christened by Willy Fowler with the name 'little bang', allows definite predictions of abundances, but without a knowledge of the overall properties of the object responsible.

\section{Properties of Supermassive Stars and Disks}

We shall first discuss some properties of supermassive stars and disks, which are two extreme examples of equilibrium configurations. A major reason for also considering highly flattened bodies is the fact that supermassive stars require much more entropy for support than is contained in interstellar gas. In order to have available definite

* Supported in part by the National Science Foundation (GP-9621).

** Alfred P. Sloan Foundation Fellow. 
models, both the angular velocity $\Omega$ and entropy $S$ are assumed to be uniform throughout the body. The assumption of uniform entropy is generally believed to be valid due to convection, at least for the stars, while the presence of magnetic fields or turbulent viscosity might maintain uniform rotation in some instances. In any case, we shall be mainly interested in the qualitative aspects of the bodies' structure and evolution, which should not depend strongly on these assumptions. (The stability of the disks may depend strongly on the rotation law, however.) Since we are interested in making helium, we take the initial composition to be mostly hydrogen.

A comparison of the properties of these two types of massive objects is presented in Table I. (For more details see Wagoner, 1969a; Bardeen and Wagoner, 1969;

\section{TABLE I}

Properties of supermassive objects $\left(10^{4} \lesssim M / M_{\odot} \equiv \mu \lesssim 10^{12} ; \Omega, S=\right.$ const.; $X_{H}=1 ; L=$ local luminosity. $)$

\begin{tabular}{|c|c|c|c|c|c|}
\hline Property & Star & & Disk & & $f_{n}(1)$ \\
\hline$\gamma=Z_{c} /\left(1+Z_{c}\right)$ & $\lesssim 0.012+2.86 \mu^{-1 / 2}$ & & $\leqslant 1$ & & \\
\hline $2 G M / R c^{2}$ & $\simeq(4 / 9) \gamma$ & $\mathrm{a}$ & $=(8 / 3 \pi) \gamma f_{1}(\gamma)$ & & 0.78 \\
\hline$G M^{2} / c J$ & $\gtrsim 10.5 \gamma^{1 / 2}$ & & $=(10 / 3 \pi) \gamma^{1 / 2} f_{2}(\gamma)$ & & 0.94 \\
\hline$E_{b} / M_{0} c^{2}$ & $\lesssim\left(1.43 \mu^{-1 / 2}+3.0 \times 10^{-3}\right)^{2}$ & & $=(1 / 5) \gamma f_{3}(\gamma)$ & & 1.85 \\
\hline Period (yr.) & $\gtrsim 1.6 \times 10^{-11} \mu \gamma^{-3 / 2}$ & & $=2.3 \times 10^{-12} \mu \gamma^{-3 / 2} f_{4}(\gamma)$ & & 0.85 \\
\hline$\left(L / L_{\odot}\right) /\left(M / M_{\odot}\right)$ & $=3.2 \times 10^{4}$ & a & $=3.2 \times 10^{4}$ & a & \\
\hline$\left(T_{9}\right)_{c}$ & $=8.83 \times 10^{3} \mu^{-1 / 2} \gamma$ & a & $=7.12 \times 10^{3} \mu^{-1 / 2} \gamma f_{5}(\gamma)$ & & 0.82 \\
\hline$T_{e}(K)$ & $=2.68 \times 10^{5}\left(T_{9}\right)_{c}^{1 / 2}$ & a & $=5.42 \times 10^{5}\left(T_{9}\right)_{c}{ }_{c}^{1 / 2}\left(\sigma / \sigma_{c}\right)^{1 / 4}$ & a & \\
\hline $\begin{array}{l}\text { Entropy } \\
\left(\operatorname{erg} K^{-1} g^{-1}\right)\end{array}$ & $S_{s}=7.76 \times 10^{7} \mu^{1 / 2}$ & a & $6.7 \times 10^{8}<S_{d}<S_{8}$ & a & \\
\hline$W / R$ & $\simeq 0.7-1.0$ & & $=0.20\left(S_{d} / S_{s}\right) f_{6}(\gamma)$ & & 0.96 \\
\hline$\beta=P_{g} / P \ll 1$ & $=8.56 \mu^{-1 / 2}$ & a & $=8.56\left(S_{\delta} / S_{d}\right) \mu^{-1 / 2}$ & a & \\
\hline$Q\left(\mathrm{~g} \mathrm{~cm}^{-3}\right)$ & $=1.30 \times 10^{5} \mu^{-1 / 2} T_{9}{ }^{3}$ & a & $=1.30 \times 10^{5}\left(S_{\delta} / S_{d}\right) \mu^{-1 / 2} T_{9}^{3}$ & $\mathrm{a}$ & \\
\hline
\end{tabular}

a Relations also valid for differential rotation

Salpeter and Wagoner, 1971.) Most properties can be expressed in terms of the relativity parameter $\gamma$ (or $Z_{c}=$ central redshift) and mass parameter $\mu=M / M_{\odot}$. The relativity parameter is limited mainly by the onset of equatorial mass 'shedding' for stars and by the development of an event horizon for the disks, if they remain stable up to that point. The relativistic functions $f_{n}(\gamma)$ vary from unity at $\gamma=0$ to their limiting values indicated. Subscript $c$ indicates the central value, $\sigma$ is the proper surface density of mass, and the binding energy $E_{b}=\left(M_{0}-M\right) c^{2}$, where $M_{0}$ is the rest mass. The period indicated is the rotation period.

We shall only consider disks in which, like the stars, radiation pressure is dominant $(\beta \ll 1)$. It is seen that the entropy in interstellar gas $\left(S \sim 10^{10} \mathrm{erg} \mathrm{K}^{-1} \mathrm{~g}^{-1}\right)$ is sufficient to guarantee this condition. It then follows that the luminosity of a star and a disk of the same mass are equal. The ratio of polar to equatorial radius, $W / R$, is seen to 
be proportional to the entropy. Of importance for nucleosynthesis is the fact that the central temperature $\left(T_{9}\right)_{c}$ (in units of $10^{9} \mathrm{~K}$ ) is approximately the same function of $\mu$ and $\gamma$ for both objects.

The evolution of supermassive stars is limited by both the 'shedding' of kinetic energy from the equator for bodies with angular momentum $J \gtrsim c^{-1} G M^{2}$, and by the development of the general relativistic collapse instability for $J \lesssim c^{-1} G M^{2}$, as is seen in Figure 1. The solid lines represent evolutionary tracks of constant angular momentum for the two masses illustrated. The presence of rotation allows much larger fractional binding energies $E_{b} / M_{0} c^{2}$ to be achieved than would otherwise be possible for the larger masses $M \gg 10^{4} M_{\odot}$. (We neglect any contribution of turbulent kinetic energy to the binding energy.)

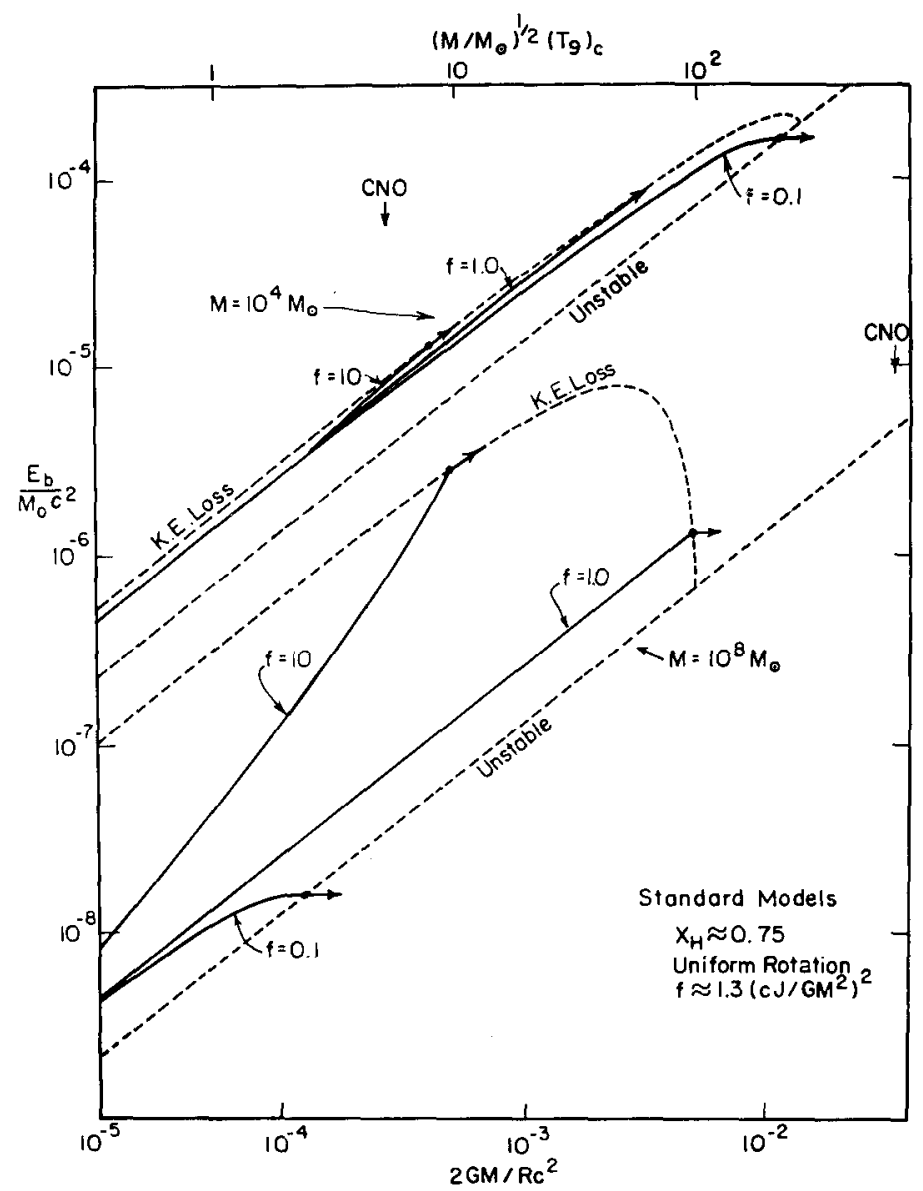

Fig. 1. Evolutionary paths of two supermassive stars with various angular momenta $J$. Also shown is the region where such stars are unstable against gravitational collapse and the region where rotation is too rapid to retain mass at the equator. The point where hydrogen burning with a normal abundance of CNO nuclei will commence is also indicated for each mass. (Adapted from

Figure 1 of Wagoner, 1969a.) 


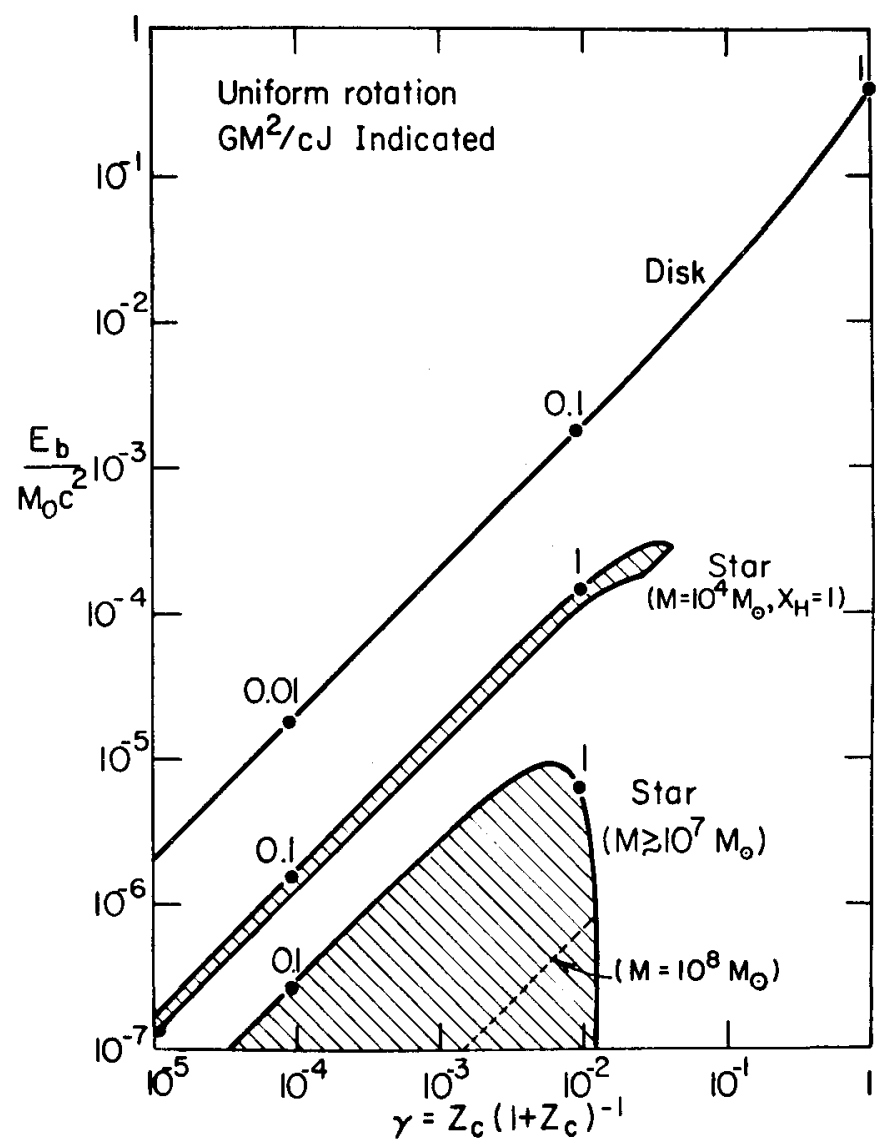

Fig. 2. Comparison of the fractional binding energies of supermassive stars and thin (low entropy) disks as a function of central redshift $Z_{c}$. The upper boundary of the domain of existence of supermassive stars (determined by equatorial mass loss) is indicated for $M=10^{4} M_{\odot}$ and $M \gtrsim 10^{7} M_{\odot}$, while the lower boundary (determined by general-relativistic instability or $J=0$ ) is indicated for $M=10^{4} M_{\odot}$ and $M=10^{8} M_{\odot}$.

Figure 2 indicates the domains of equilibrium models of stars and disks. Note that a star on the verge of shedding and a disk with the same value of $G M^{2} / c J$ have relativity parameters differing by a factor of $\sim 10^{2}$. A disk can therefore reach central temperatures $\sim 10^{2}$ times larger than a star of the same mass and angular momentum. The corresponding binding energy is also seen to be much larger for the disk, due to the removal of radiation pressure as the major supporting force. We shall not consider the evolution of supermassive stars after shedding commences, which has been investigated by Bisnovatyi-Kogan et al. (1967) but is complicated by many factors.

The rate of change of the central temperature of a supermassive star of fixed rest mass $M_{0}$ will be mainly governed by the loss of photons, characterized by the KelvinHelmholtz time scale $\tau_{K}=\Delta E_{b} / L$. Although the central temperature of the very mas- 
sive stars can also increase due to loss of angular momentum, this is unlikely to occur on a time scale less than the Kelvin-Helmholtz time of $\tau_{K} \lesssim 3 \times 10^{3} \mathrm{yr}$ for these masses $M \gtrsim 10^{7} M_{\odot}$. If the star reaches a point where hydrogen burning can commence, the central temperature will remain approximately constant for a time $\tau_{N}=E\left(4 p \rightarrow{ }^{4} \mathrm{He}\right) / L$ $\approx 3 \times 10^{6} \mathrm{yr}$.

The situation is quite different for the disks, in which the loss of photons only leads to a flattening of the disk at constant temperature for a time $\tau_{K}=\Delta E_{b} / L<E_{b} / L$ until enough entropy is lost so that the gas pressure begins to dominate, at which point the temperature begins to decrease (Salpeter and Wagoner, 1971). On the other hand, the loss of angular momentum by a disk of fixed rest mass will increase the temperature with some time scale $\tau_{J}$. However, unlike the situation in the stars, the rise in temperature will not necessarily halt when nuclear burning commences, since the radial collapse is unaffected by the luminosity.

The implications of these properties for helium production will now be explored separately for supermassive stars and disks. It is encouraging that there appears to be increasing evidence of the presence of very massive, magnetized, rotating bodies in quasi-stellar objects and active galactic nuclei (Kinman et al., 1968; Morrison, 1969; Cavaliere et al., 1969; Visvanathan, 1970).

\section{Helium Production by Supermassive Stars}

The factors affecting the conversion of hydrogen to helium within supermassive stars are presented in Figure 3. Recall that the maximum mass which can reach a given central temperature $\left(T_{9}\right)_{c}$ is determined by the onset of equatorial shedding and/or gravitational collapse. Plotted are the inverses of the average inverse mean lifetimes throughout stars of various masses of protons due to the $p-p$ reaction and CNO bi-cycle and of helium due to the triple-alpha reaction, multiplied by the appropriate mass fraction. Also included is the effective surface temperature, indicating emission in the ultraviolet during hydrogen burning.

Consider a pure hydrogen star undergoing Kelvin-Helmholtz contraction. The mass fraction of ${ }^{4} \mathrm{He}$ formed if it reaches temperatures $\left(T_{9}\right)_{c}<1$ will be

$$
\mathrm{X}_{\alpha} \approx \tau_{K} /\left\langle\tau_{p}(p p)\right\rangle \text {. }
$$

Using the maximum values of $\tau_{K}$ indicated on the Figure, it is seen that $\mathrm{X}_{\alpha} \ll 1$ for this range of temperature. However, the triple- $\alpha$ reaction will then come into play, converting some of the helium into a mass fraction

$$
X\left({ }^{12} \mathrm{C}\right) \approx \tau_{K} X_{\alpha} /\left\langle\tau_{\alpha}(3 \alpha)\right\rangle
$$

of carbon. This in turn will initiate the operation of the CNO cycle, which requires very little carbon in order that the evolution be halted for a time

$$
\left\langle\tau_{p}(\mathrm{CNO})\right\rangle \approx E\left(4 p \rightarrow{ }^{4} \mathrm{He}\right) / L \sim 3 \times 10^{6} \mathrm{yr} .
$$

The solution of Equations (1), (2), and (3) leads to $\mathrm{X}_{\alpha} \approx 10^{-4}, \mathrm{X}\left({ }^{12} \mathrm{C}\right) \approx 10^{-11}$ $10^{-10}$, and $\left(T_{9}\right)_{c} \approx 0.3-0.5$ at the commencement of hydrogen burning for masses 


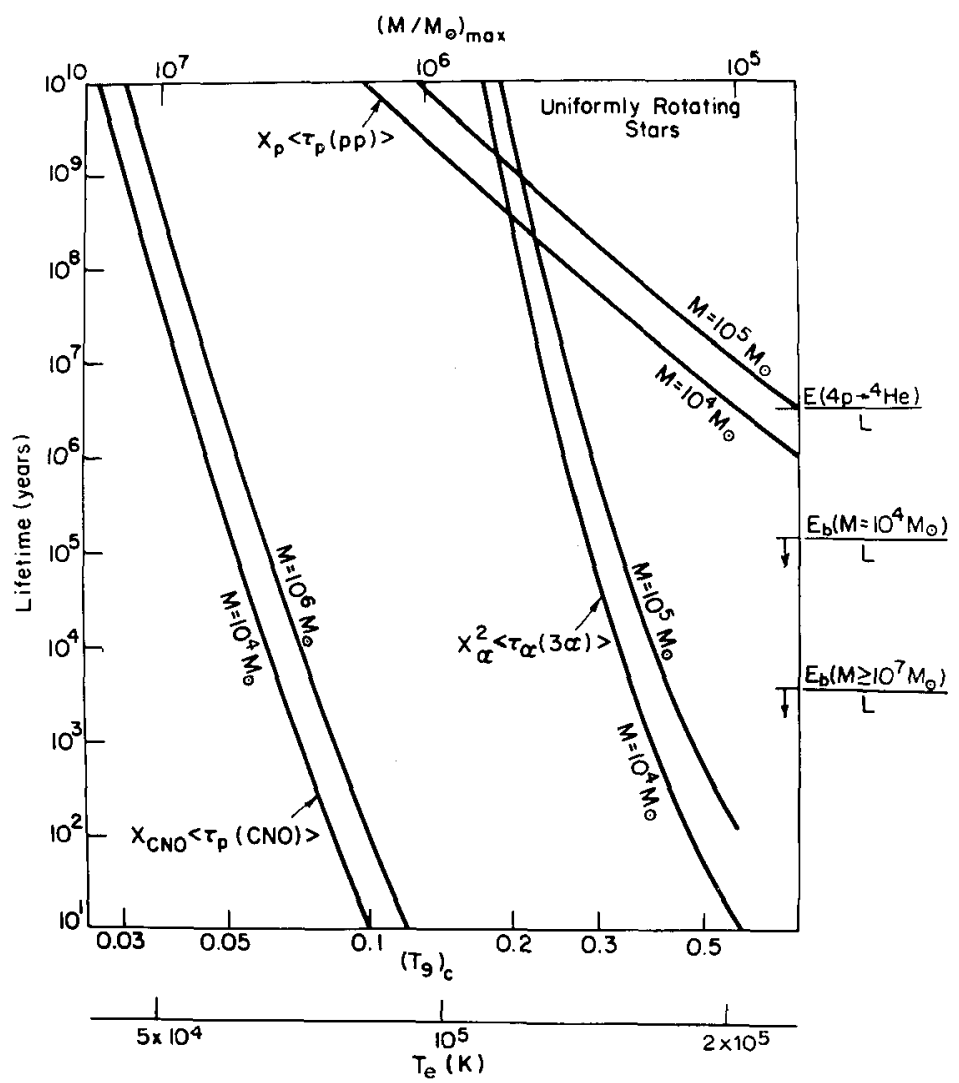

Fig. 3. Various lifetimes affecting the production of helium in supermassive stars. Also indicated are the effective surface temperature and maximum mass corresponding to each value of central temperature $\left(T_{9}\right)_{c}$.

$M / M_{\odot} \approx 10^{4}-10^{5}$ (extrapolating the results of Boury (1963)). The main result is therefore that initially pure hydrogen stars of masses $M \gtrsim 2 \times 10^{5} M_{\odot}$ cannot reach high enough temperatures to initiate significant conversion of hydrogen to helium.

If the star is fortunate enough to begin life with a Population I abundance of CNO, $\mathrm{X}_{\mathrm{CNO}} \approx 10^{-2}$, then it is seen from Equation (3) and Figure 3 that the mass limit is raised to $M \approx 3 \times 10^{6} M_{\odot}$, as first discussed by Fowler (1966a) and Roxburgh (1965). Even a Population II amount, $\mathrm{X}_{\mathrm{CNO}} \approx 10^{-4}$, lowers the mass limit only slightly to $M \approx 2 \times 10^{6} M_{\odot}$.

The burning will usually convert most of the $\mathrm{CNO}$ nuclei to ${ }^{14} \mathrm{~N}$ while producing helium. If nothing else happens, the star will subsequently evolve to a temperature where helium burning will commence. The net result will then be little helium production unless the helium burning or subsequent nuclear processes lead to a nuclear explosion before much helium has been consumed.

However, it is likely that something else will happen, since supermassive stars are known to be pulsationally unstable (Ledoux, 1941; Schwarzschild and Härm, 1959; 
Osaki, 1966). Osaki studied stars of mass $10^{4} \leqslant M / M_{\odot} \leqslant 2 \times 10^{5}$, and found that the time scale of pulsational amplitude increase was $\tau_{P} \approx 2(v+3)^{-1} \tau_{K}$ for a nuclear energy source $\propto T^{\nu}$. Under the assumption that the gain in pulsational energy is balanced by the energy lost through mass ejection for each cycle, he found that the mass-loss time scale is also of the order of $\tau_{K}$. Since $\tau_{K} \ll \tau_{N} \approx 3 \times 10^{6} \mathrm{yr}$, most of the mass ejected would have a small helium abundance, $\mathrm{X}_{\alpha} \approx \tau_{K} / \tau_{N} \lesssim 10^{-2}$. Although Osaki ignored the effects of rotation and general relativity, it appears that the inclusion of these factors will not change these results drastically. However, some recent investigations arrive at somewhat different estimates for the effectiveness of pulsational mass-loss (Shaviv, 1970), as the discussion following Dr Kippenhahn's talk indicated.

Nevertheless, it is clear that such supermassive stars could deliver a significant amount of helium to the interstellar medium through this mechanism only if the pulsational mass-loss time scale were comparable to the hydrogen-burning time. The theory of pulsations is still sufficiently uncertain that this might be possible, although not probable.

Of course, more massive stars will reach hydrogen-burning temperatures during gravitational collapse, but only a small range of masses are able to provide enough nuclear energy to overcome the gravitational binding and expel material (Fowler, 1966b; Bisnovatyi-Kogan, 1968).

\section{Helium Production by Supermassive Disks}

The relevant factors governing helium production in disks are presented in Figure 4 in the same way as for stars in Figure 3. As previously noted, much larger masses can reach hydrogen-burning temperatures than for stars, but the effective surface temperatures are similar. The lifetimes due to the various nuclear processes are evaluated at the center of the disk. However, unlike supermassive stars, the ratio of central temperature to average temperature through the disk is not much greater than unity (Salpeter and Wagoner, 1971). The minimum lifetimes are plotted, corresponding to the gas pressure becoming comparable to the radiation pressure.

The sequences of nuclear processes possible for a disk containing various initial amounts of CNO nuclei are similar to those for a star. One difference is that the Kelvin-Helmholtz flattening time for a relativistic disk can be longer than the hydrogen-burning time. Although it is seen from Figure 4 that this time can be long enough to allow significant processing by the $p-p$ chain, under most circumstances the triplealpha reaction will then devour the helium over this period of time.

In analogy with the stellar case, we shall define the maximum hydrogen-burning mass of a disk to be such that $\tau_{p}=\tau_{N} \approx 3 \times 10^{6} \mathrm{yr}$. It is then seen from Figure 4 that the mass limit corresponding to Population I CNO abundance is $M \approx 2 \times 10^{10} M_{\odot}$, with correspondingly smaller limits for decreasing amounts of initial CNO, similarly to the situation for stars. Again, the surface temperature corresponds to emission in the ultraviolet, although it varies slowly over the surface of the disk. 


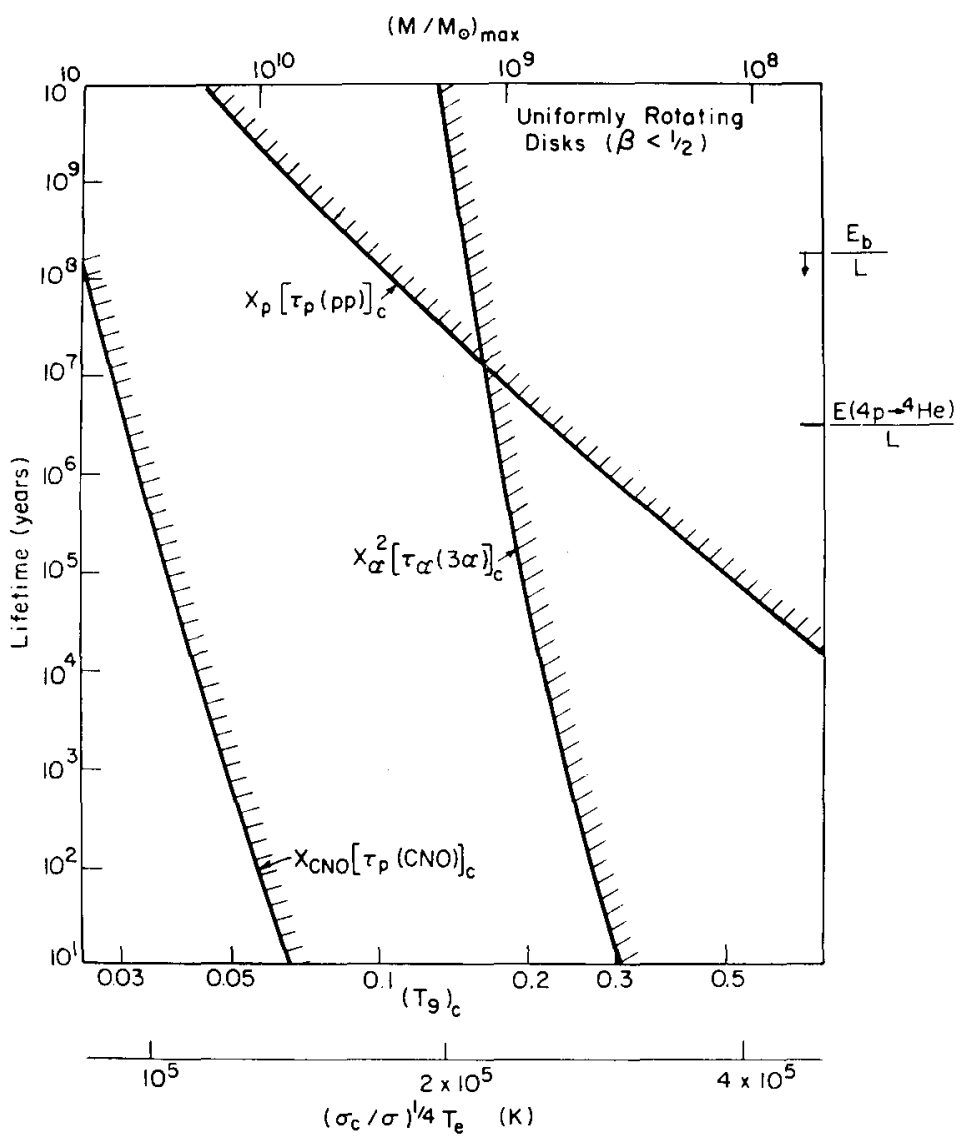

Fig. 4. Same as Figure 3 for supermassive disks.

Since disks are not susceptible to the same pulsational instability found in supermassive stars, another means must be found for delivering any helium produced into the interstellar medium. The well-known modes of instability of a disk are not effective, since there is no reason why they should only occur during hydrogen burning, which is necessary in order that helium is made but not later destroyed.

However, the evolution of the disk due to angular-momentum loss appears to provide a possibility. This is due to the previously mentioned fact that the mechanism responsible for the evolution continues to operate during nuclear burning. Thus in principle it is possible to release a large amount of energy rapidly as the temperature rises through that necessary for nuclear ignition. Two conditions which are necessary but need not be sufficient for the expulsion of matter are the following:

(a) The entropy increase due to the nuclear energy released must not be great enough to lead to a spherical equilibrium state. Using Table I, it is found that this condition is satisfied for masses $M \gtrsim 10^{6} M_{\odot}$. However, such masses are rather tightly 
bound at this stage $\left(T_{9} \sim 0.1\right)$, so that any matter expelled would usually have acquired more than its share of the available energy.

(b) The angular-momentum loss time scale must be less than $\tau_{N}$, the time required for the star to radiate the nuclear energy. This guarantees that the rise in temperature accompanying the loss of angular momentum will be fast enough to prevent the atmosphere of the disk from disposing of the energy while in equilibrium. Any expulsion of matter should occur mainly in the directions normal to the plane of the disk.

\section{Little Bangs}

We now turn to helium production through the operation of process 2 , the rapid expansion of massive objects from temperatures $>10^{10} \mathrm{~K}$. Element synthesis is computed for individual volume elements $V$ containing a fixed number of baryons, which because of the rapid expansion are taken to be thermodynamically unaffected by conditions in the rest of the body. In addition, the neutrinos are assumed to escape

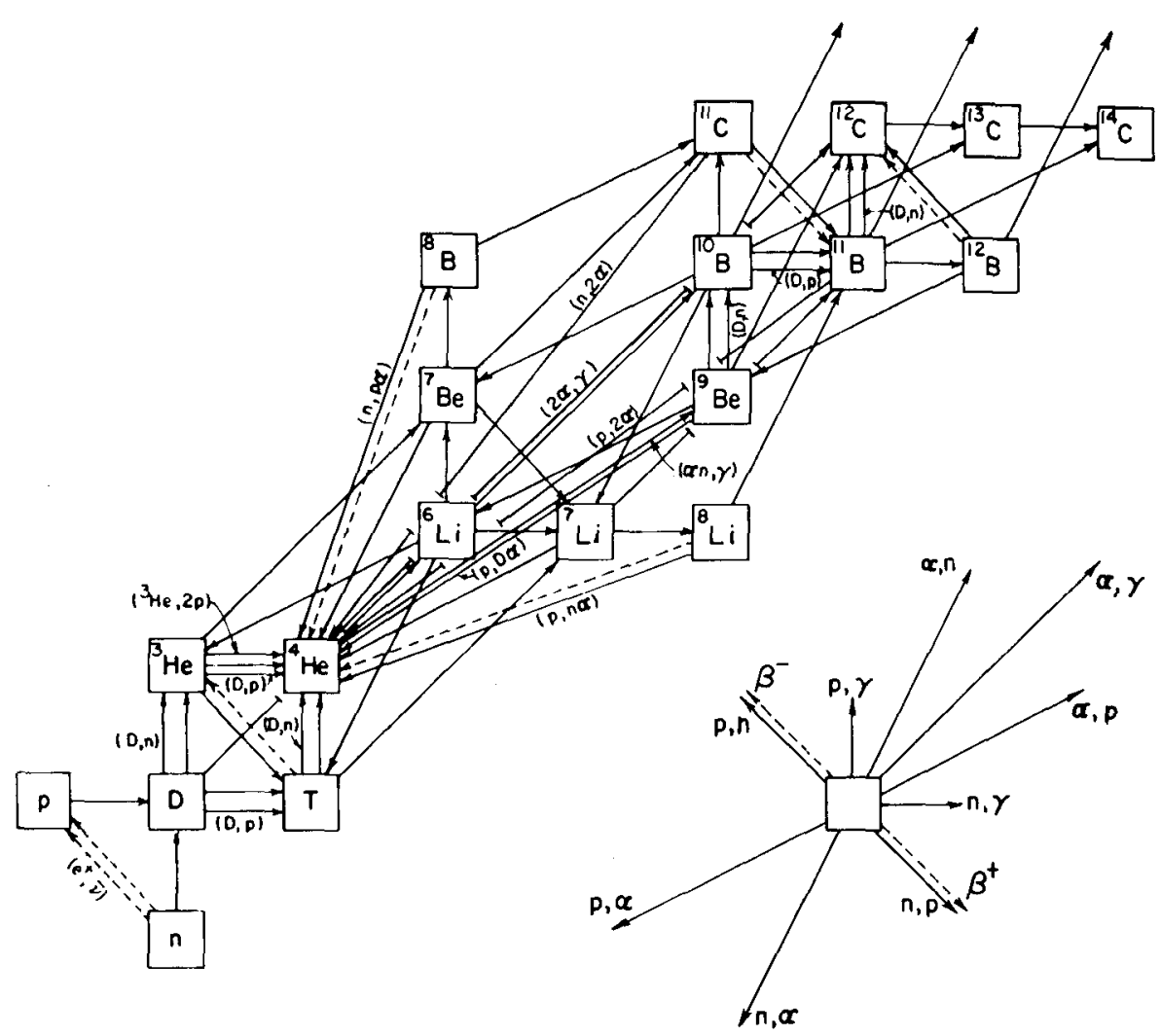

Fig, 5a. Diagram of all reactions among nuclei with $Z \leqslant 6$ (including the inverses of the strong reactions) included in the computation of element production by 'little bangs' expanding at or near the 'gravitational' rate $\sqrt{ } 24 \pi G Q$. 


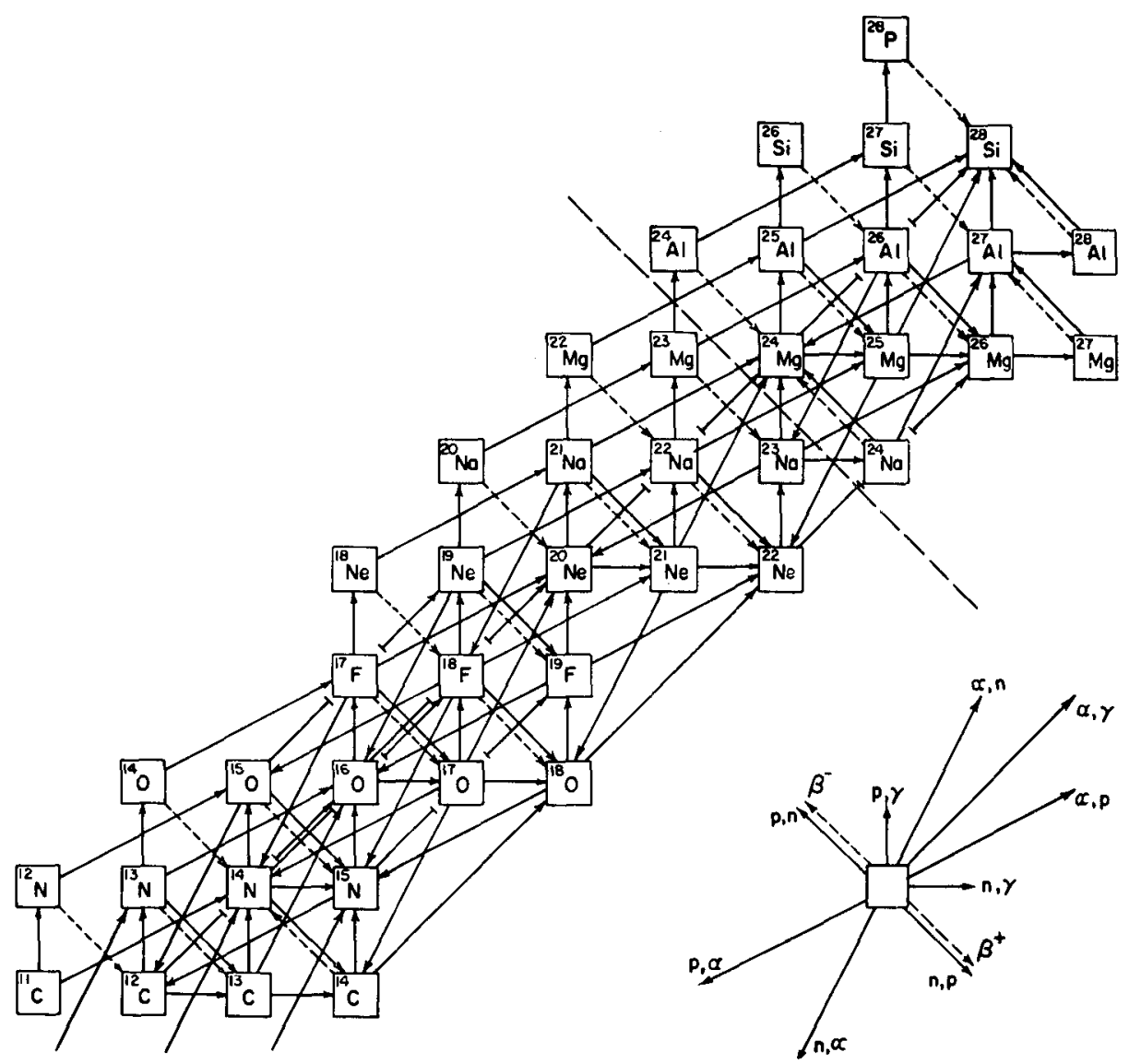

Fig. 5b. Same as Figure 5a for the remainder of the nuclei with $A \leqslant 28$. The point above which individual abundances cannot be reliably computed is also indicated by the dashed line.

freely from the body. Thus, element production within any comoving volume is affected by at most three local properties (Wagoner, 1969b):

(a) The expansion rate, taken to be proportional to the 'gravitational' rate $\sqrt{ } 24 \pi G \varrho$,

$$
V^{-1} \mathrm{~d} V / \mathrm{d} t=\xi \sqrt{ } 24 \pi G \varrho,
$$

where $\varrho$ is the total mass-energy density.

(b) The relation between baryon density $\varrho_{b}$ and temperature $T_{9}$,

$$
\varrho_{b}=h T_{9}^{3} \mathrm{~g} \mathrm{~cm}^{-3},
$$

where $h$ is constant while the expansion is adiabatic. The restriction to nondegenerate conditions implies $h<10^{5} \mathrm{~g} \mathrm{~cm}^{-3}$ for $T_{9} \gtrsim 3$.

(c) The initial neutron-proton ratio $X_{n} / X_{p}$, which must be specified unless the maximum temperature $T_{i}$ is high enough and $\xi$ small enough so that the $n-p$ weak reactions are in equilibrium. We shall consider two cases: 


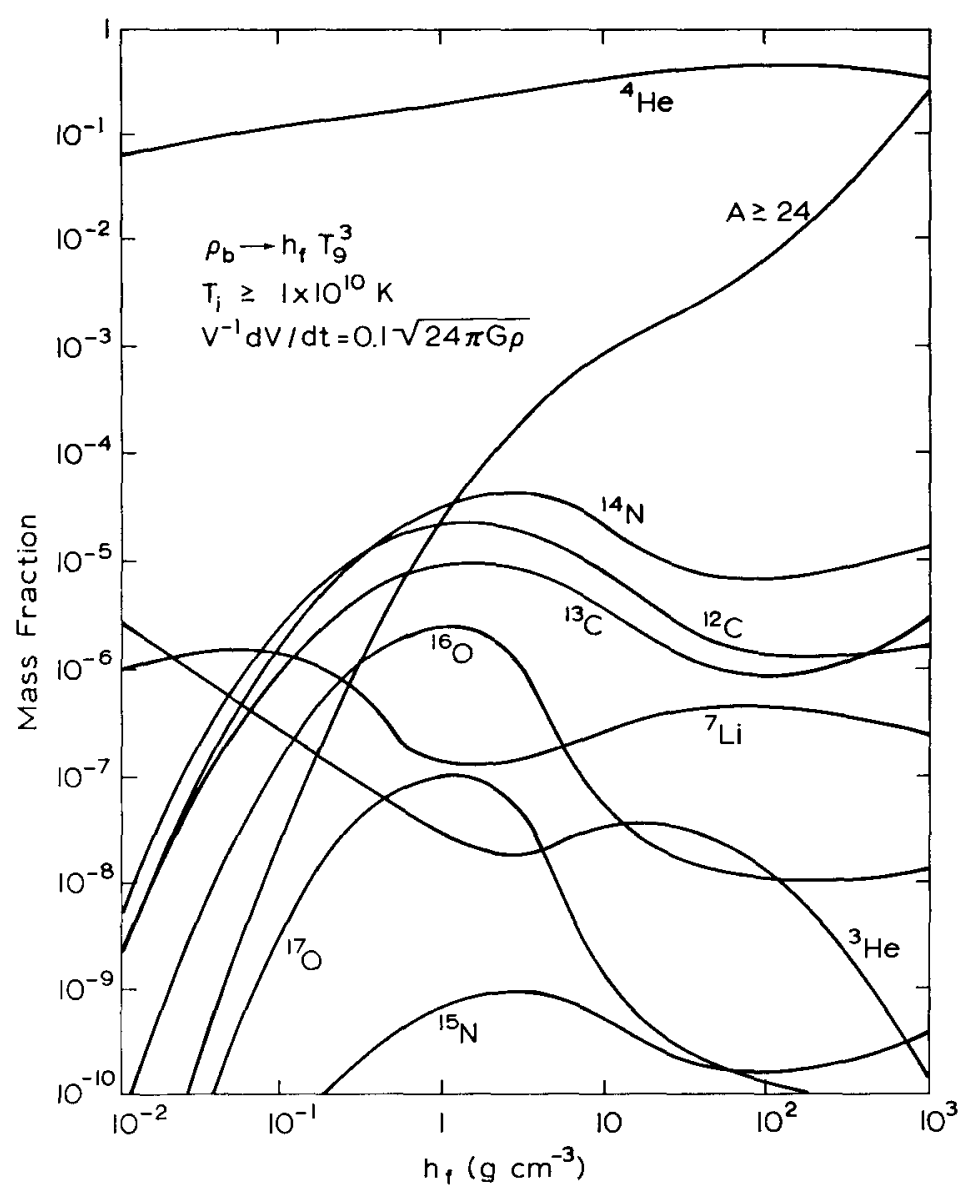

Fig. 6a. Final abundances produced by little bangs expanding from the temperature $T_{\imath}$ with $\xi=0.1$.

(i) $0.1 \leqslant \xi \leqslant 10$ with $T_{i} \geqslant 1-4 \times 10^{10} \mathrm{~K}$, so that the nucleons are initially in equilibrium.

(ii) $\xi \gtrsim 10^{4}$, with $X_{p} / X_{n}$ at $T=10^{10} \mathrm{~K}$ a free variable. At temperatures $T \leqslant 10^{10} \mathrm{~K}$, all other nuclei are strongly in equilibrium, and usually much less abundant than the nucleons.

If the expansion were preceded by the (adiabatic) collapse of a supermassive star, then masses $M \gtrsim 2 \times 10^{6} M_{\odot}$ would be within their Schwarzschild radius at $T_{9}=10$, and the value of $h$ during the final stage of the expansion (following pair annihilation and possible loss of entropy through neutrino emission, but neglecting nuclear heating) would be limited by $h_{f} \geqslant 1.3 \times 10^{5}\left(M_{\odot} / M\right)^{1 / 2}$ (see Table I). Of course, the object might also be simply emerging from a singularity (Ambartsumian, 1965) like a delayed remnant of the big bang (Novikov, 1964; Ne'eman, 1965).

The nuclear reaction network used for case (i) is shown in Figures $5 a$ and $b$, and represents an updating of the original network used by Wagoner et al. (1967) for this 


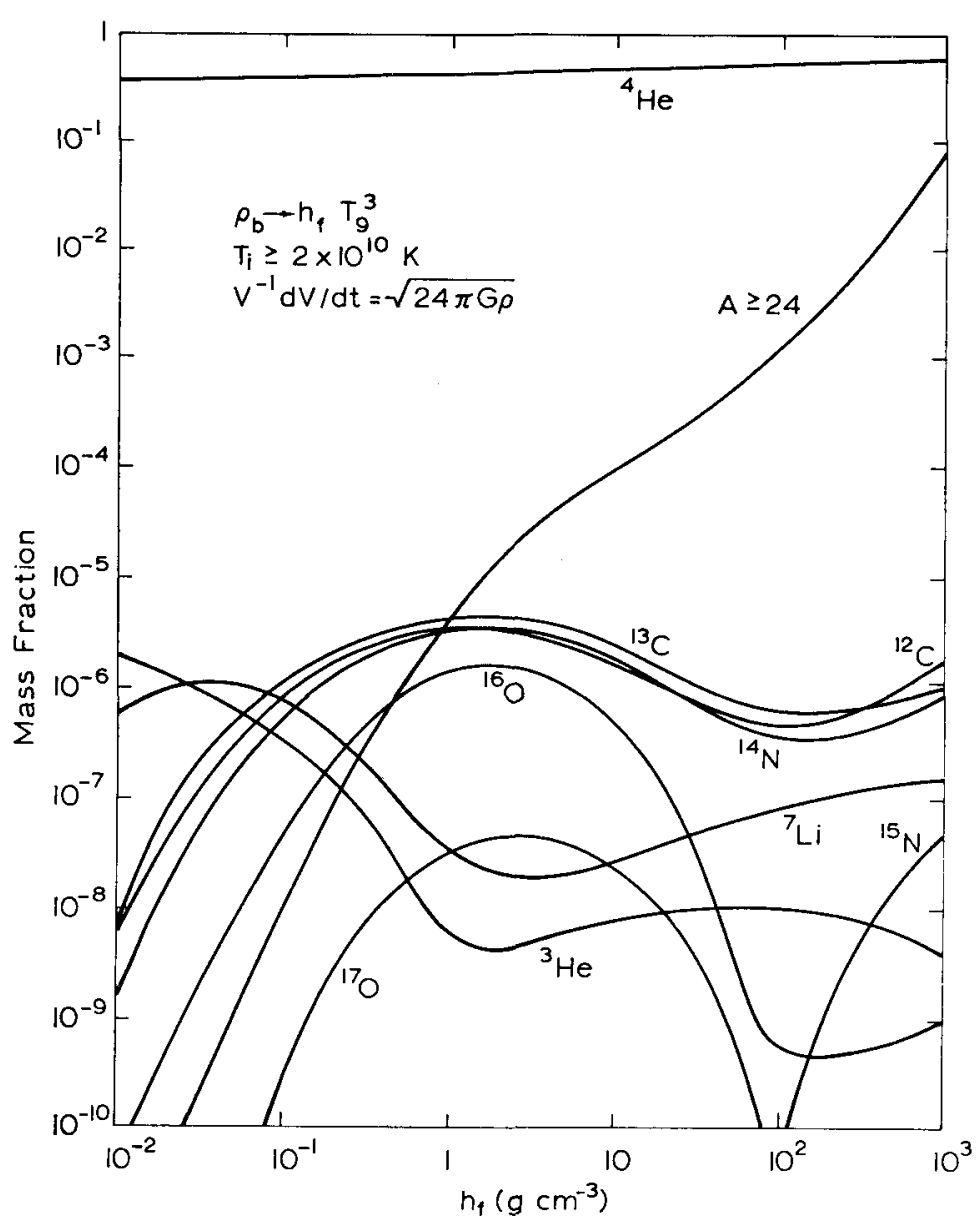

Fig. 6b. Same as Figure 6 a for $\xi=1$.

problem. Although we are here mainly interested in ${ }^{4} \mathrm{He}$, it must be determined whether the abundances of any heavier elements produced are at least consistent with observation. Since the expansion times are comparable to the neutron decay time for this case, synthesis beyond helium proceeds mainly through proton and alpha-particle reactions with the stable and slightly proton-rich unstable nuclei.

In Figures $6 \mathrm{a}, 6 \mathrm{~b}$, and $6 \mathrm{c}$ are plotted the final abundances (by mass) produced as a function of $h_{f}$ for $\xi=0.1,1$, and 10 . Figure $6 \mathrm{~b}$ represents the same situation considered by Wagoner et al. (1967), except for the inclusion of neutrino emission. Note that the higher values of $h_{f}$ lead to unacceptably large amounts of the very heavy elements $(A \geqslant 24)$, which are probably produced in the region of the iron group. Also, the amount of CNO nuclei produced is never larger than that of extreme Population II, and the ratio ${ }^{13} \mathrm{C} /{ }^{12} \mathrm{C}$ is higher than the upper limits determined in some Population II stars (Cohen and Grasdalen, 1968). Nevertheless, the results for the lower 


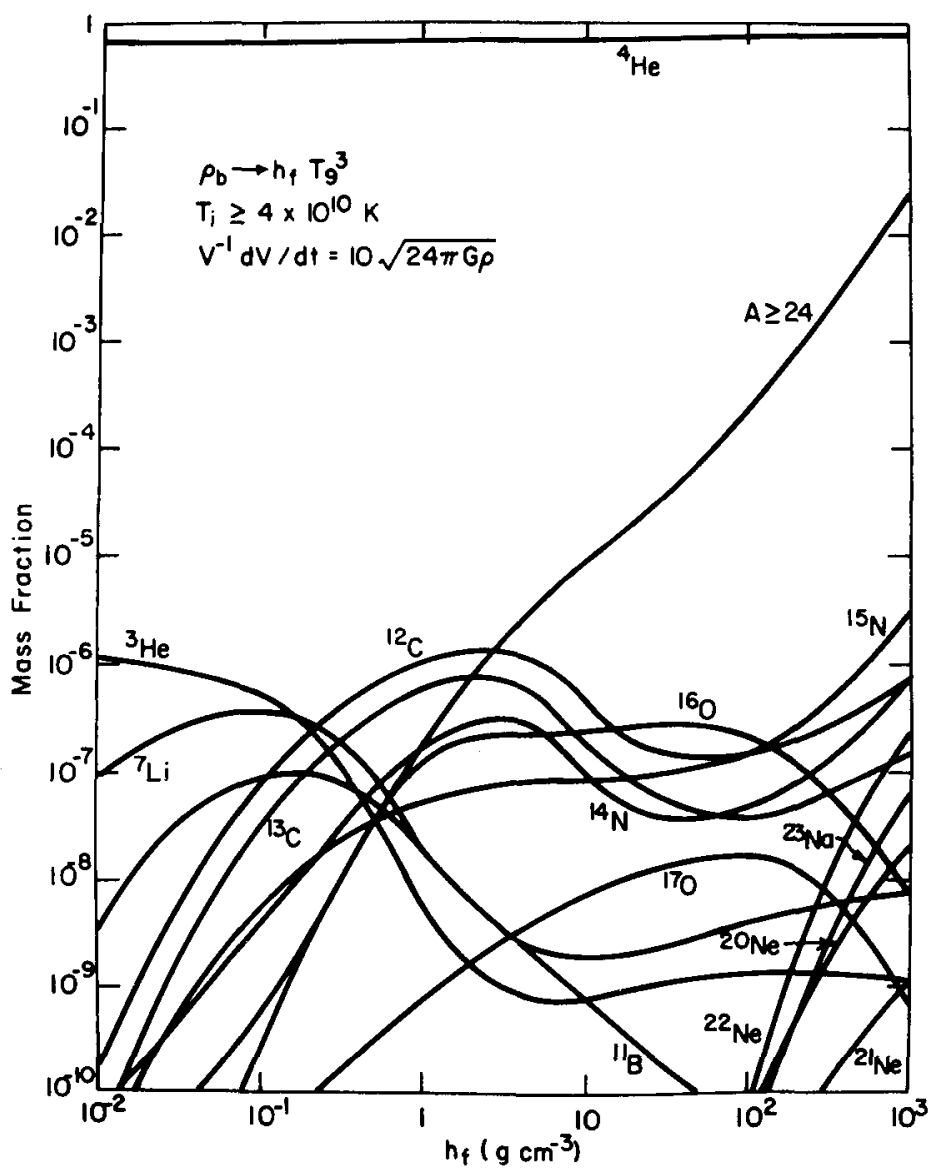

Fig. 6c. Same as Figure 6a for $\xi=10$.

$h_{f}$ values are still consistent with observation, with the possible exception of the relatively large amount of ${ }^{7} \mathrm{Li}$ produced. It must be remembered that subsequent mixing with an interstellar medium of pure hydrogen would reduce all these abundances.

The results for ${ }^{4} \mathrm{He}$ alone are presented in Figure 7. The amount of helium produced depends most strongly on the expansion rate, which determines at which temperature the equilibrium neutron-proton ratio of

$$
\mathrm{X}_{n} / \mathrm{X}_{p}=\exp \left(-15.0 / T_{9}\right)
$$

'freezes out'. Except for some subsequent neutron decay, this ratio determines the final ${ }^{4} \mathrm{He}$ abundance, since virtually all the neutrons available are used in making ${ }^{4} \mathrm{He}$ when the temperature has fallen to $T_{9}=1-7$, depending on $h_{f}$.

The helium abundance produced in expansions with $\xi \approx 1$ is of the order of that 


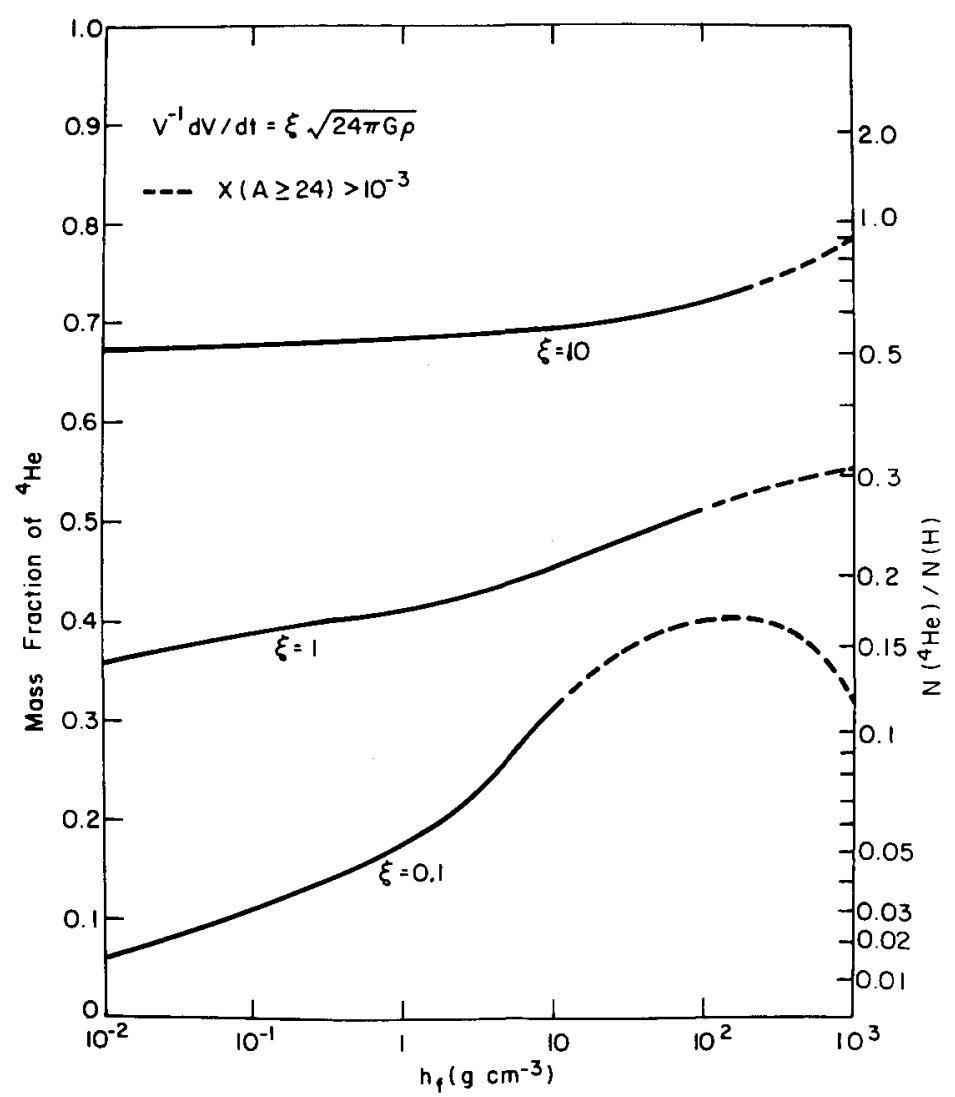

Fig. 7. Final abundances of ${ }^{4} \mathrm{He}$ produced by the little bangs indicated in Figures $6 \mathrm{a}, \mathrm{b}, \mathrm{c}$. The dashed portion of the curves indicates those values of the parameters $\xi$ and $h_{f}$ which lead to an unexceptably large amount of very heavy elements.

observed in Population I objects. For lower values of $h_{f}$, the abundance is only slightly higher than that produced in the 'standard' big bang, the difference being due mainly to the lack of neutrino interactions with the nucleons.

Turning now to case (ii), it was found by Wagoner (1969b) that abundances resembling those in the most metal-deficient stars could be produced by very rapid expansions $\left(\xi \gtrsim 10^{4}\right)$ from high temperatures if $\mathrm{X}_{p} / \mathrm{X}_{n}=1.00 \pm 10^{-3}$ at $T_{9}=10$, before element production. Due to the rapid expansion this ratio is preserved during the expansion, even while the nucleons are depleted in the production of virtually pure ${ }^{4} \mathrm{He}$. The final abundances produced by two typical sets of parameters are compared with solar system abundances in Figure 8, taken from the paper of Wagoner (1969b). The most encouraging feature is the agreement of many of the isotopic ratios. However, the point of major interest here is that neutron-proton equality at high temperatures is possible to achieve naturally in a number of ways (see Wagoner $(1969 \mathrm{~b})$ and case (i) for $\xi \gg 1$ ), so that explosions of essentially pure helium are possible. 


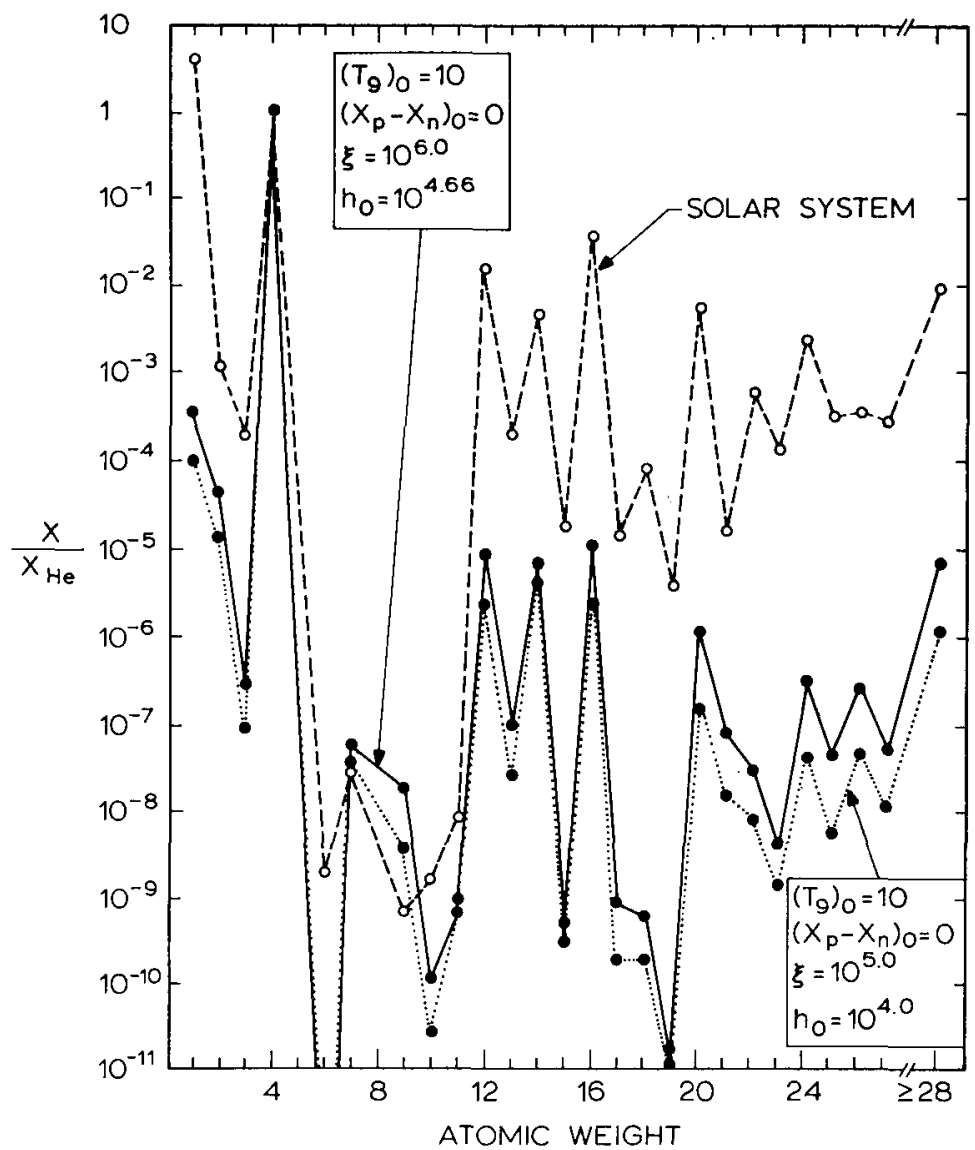

Fig. 8. A comparison of the abundances produced by two very rapid little bangs with those observed in the vicinity of the solar system. These models are characterized by values of $h_{0}\left(=h\left(T_{9}=10\right)\right)$ which give nearly the maximum production of the CNO group without violating the observational condition $\mathrm{X}(\mathrm{CNO}) \gtrsim \mathrm{X}(A \geqslant 28)$. The abundances have been normalized to that of ${ }^{4} \mathrm{He}$, since very little hydrogen is produced. (Reproduced from Figure 10 of Wagoner, 1969b).

\section{Conclusions}

The major results of this investigation may be summarized as follows:

(1) Uniformly rotating supermassive stars of mass $M \lesssim 2 \times 10^{5} M_{\odot}$ can convert pure hydrogen into helium. The corresponding range for an initially normal abundance of carbon, nitrogen, or oxygen is $M \lesssim 3 \times 10^{6} M_{\odot}$. However, the star will be able to deliver a significant amount of helium into interstellar space only if the mass loss time scale due to its pulsational instability is of the same order as the nuclear burning time, $3 \times 10^{6} \mathrm{yr}$, which appears unlikely, or if subsequent nuclear processes lead to an explosion before a significant amount of helium has been consumed.

(2) Uniformly rotating supermassive disks of mass $M \lesssim 3 \times 10^{9} M_{\odot}$ can convert pure hydrogen into helium. The corresponding range for an initially normal abun- 
dance of carbon, nitrogen, or oxygen is $M \lesssim 2 \times 10^{10} M_{\odot}$. If instabilities do not fragment the disk, the loss of angular momentum may lead to expulsion of material during hydrogen burning.

(3) Little bangs expanding from temperatures $T \gtrsim 10^{10} \mathrm{~K}$ at rates of the order of the gravitational rate $V^{-1} \mathrm{~d} V / \mathrm{d} t=\sqrt{ } 24 \pi G Q$ produce helium mass fractions only slightly greater than those in the corresponding big-bang model. In no case is it possible to produce many other elements with Population I abundancs, however.

\section{Acknowledgements}

The author wishes to thank E. E. Salpeter, W. D. Arnett, G. Shaviv, and F. Pacini for helpful discussions.

\section{References}

Ambartsumian, V. A.: 1965, in The Structure and Evolution of Galaxies, 13th Solvay Conference, Interscience Publishers, New York.

Bardeen, J. M. and Wagoner, R. V.: 1969, Astrophys. J. 158, L65.

Bisnovatyi-Kogan, G. S.: 1968, Astron. Zh. 45, 74 (Engl, transl. Soviet Astron.-AJ 12, 58).

Bisnovatyi-Kogan, G. S., Zel'dovich, Ya, B., and Novikov, I. D.: 1967, Astron. Zh. 44, 525 (Engl. transl. Soviet Astron.-AJ 11, 419).

Boury, A.: 1963, Ann. Astrophys. 26, 354.

Cavaliere, A., Pacini, F., and Setti, G.: 1969, Astrophys. Letters 4, 103.

Cohen, J. G. and Grasdalen, G. L.: 1968, Astrophys. J. 151, L41.

Fowler, W. A.: 1966a, Astrophys. J. 144, 180.

Fowler, W. A.: 1966b, in High-Energy Astrophysics, Proc. Course 35 Intern. Sch. Phys. "Enrico Fermi' (ed. by L. Gratton), Academic Press, New York.

Hoyle, F. and Fowler, W. A.: 1963a, Monthly Notices Roy. Astron. Soc. 125, 169.

Hoyle, F. and Fowler, W. A.: 1963b, Nature 197, 533.

Kinman, T. D., Lamla, E., Ciurla, T., Harlan, E., and Wirtanen, C. A.: 1968, Astrophys. J. 152, 357. Ledoux, P.: 1941, Astrophys. J. 94, 537.

Morrison, P.: 1969, Astrophys. J. 157, L73.

Ne'eman, Y.: 1965, Astrophys. J. 141, 1303.

Novikov, I. D.: 1964, Astron. Zh. 141, 1075 (Engl. transl. Soviet Astron.-AJ 8, 857).

Osaki, Y.: 1966, Publ. Astron. Soc. Japan 18, 384.

Roxburgh, I. W.: 1965, Nature 207, 363.

Salpeter, E. E. and Wagoner, R. V.: 1971, Astrophys. J. 164, 557.

Schwarzschild, M. and Härm, R.: 1959, Astrophys. J. 129, 637.

Shaviv, G.: 1970, private communication.

Visvanathan, N.: 1970, private communication.

Wagoner, R. V.: 1969a, Ann. Rev. Astron. Astrophys. 7, 553.

Wagoner, R. V.: 1969b, Astrophys. J. Suppl. No. 162, 18, 247.

Wagoner, R. V. and Salpeter, E. E.: 1970, Paper presented at IAU Symposium No. 44, External Galaxies and Quasi-Stellar Objects.

Wagoner, R. V., Fowler, W. A., and Hoyle, F.: 1967, Astrophys. J. 148, 3.

\section{DISCUSSION}

A. Underhill: Where do you expect these extremely massive objects to appear in the universe?

$R$. Wagoner: If they exist, massive objects would most likely be found in the active nuclei of galaxies as well as QSO's (which may be the same thing). Of course, many supermassive stars and/or disks may have existed in galaxies during their formation. Another possibility is that a young galaxy is a little bang emerging from a 'singularity'. 
J. Pachner: As long as the dimensionless parameter $|\Omega|{ }^{2} / 4 \pi G Q,|\Omega|$ being the angular velocity, $G$ the Newtonian constant of gravitation, and $\varrho$ the mass density, is much smaller than unity the influence of rotation may be fully neglected. As soon as the parameter is comparable to or is greater than unity, the influence of rotation becomes very important from the point of view of general relativity. In this case the assumption of uniform rotation can give us results, especially concerning stability, which differ essentially from those when differential rotation is taken into account. 